\title{
The Bluetooth Technology: State of the Art and Networking Aspects
}

\author{
Dajana Cassioli, Andrea Detti, Pierpaolo Loreti, Franco Mazzenga, and \\ Francesco Vatalaro \\ University of Rome Tor Vergata, \\ Dipartimento di Ingegneria Elettronica \\ and \\ RadioLabs \\ Consorzio Università Industria - Laboratori di Radiocomunicazioni \\ \{surname\}@ing. uniroma2. it
}

\begin{abstract}
Bluetooth is considered as a low-cost short-range wireless technology to provide communication functionalities, ranging from wire replacement to simple personal area network. In Bluetooth local networking applications a critical issue still under study is the evaluation of the network capacity when multiple piconets are simultaneously active in the same area, while providing mutual interference. In this paper we first provide a review of the main characteristics of the Bluetooth technology then we propose a semi-analytical approach to calculate the packet loss probability, and the aggregate network throughput. The analytical approach was validated by extensive comparison with simulation results showing a good agreement.
\end{abstract}

\section{Introduction}

The increasing importance of Internet web-based data applications and the pressing request for mobility pushed the research activities towards the definition of new global radio access networks.

Wireless personal area networks (PANs) represent the first access level to the global network. An equipment used for PAN communications should be low-cost, should provide communications among very different appliances, should interface with both wired and wireless external networks and should assure a relatively large traffic capacity. In a typical domestic or office environment the number of communicating appliances and/or terminals accessing to Internet can be quite large and their position cannot be easily predicted. Therefore a proper selection of a suitable wireless technique to connect them is mandatory. Furthermore to ensure full connectivity, this radio technology should be able to dynamically create and to manage ad hoc network(s) among the communicating terminals in the area.

The Bluetooth technology [1]-6] is conceived as an effective low-cost solution to the many problems of PAN communications. In this paper we first provide a review of the main features of the Bluetooth technology and a brief discussion on 
the characteristics of the currently available market products. Then we obtain a closed form solution for the packet loss probability in a Bluetooth network composed of uncoordinated and interfering piconets. The formulation is valid under many different operating conditions and it is used to evaluate the overall network throughput. The paper is organized as follows. In Section 2 we provide a brief description of the main features of the Bluetooth technology and in Section 3 we summarize the main characteristics of the Bluetooth chip-modem currently available on the market. In Section 4 we obtain the packet loss probability and in Section 5 we define the Bluetooth network throughput. In Section 6 we validate the proposed approach comparing the theoretical results with simulation results. Finally, in Section [7 we draw our conclusions.

\section{Bluetooth Technology}

The Bluetooth was conceived to connect heterogeneous pieces of equipment, such as cellular phones, portable PCs, printers and, in the near future, domestic appliances, both among them and with external networks (mainly the Internet). These equipment and appliances contain a Bluetooth modem, commonly implemented as (possibly) low cost chip(s).

Three main fields of applications of Bluetooth technology have been identified:

- cable replacement among different appliances (point-to-point applications);

- access points to the Internet and to other external networks, both wired and wireless (point-to-multipoint applications);

- personal area networks.

The cable replacement application will render easier the interchange of data among different peripherals. Some examples are: the wireless connection between a cellular phone and the earphone, the connection of a mouse and a keyboard with the PC processing unit, etc. Since Bluetooth is intended as a de-facto standard, its adoption for cable replacement overcomes many problems related to cable standardization worldwide.

Bluetooth can be used to implement very low cost wireless access points, providing secure channels over which many transactions (information retrieval, automatic payment, etc.) can occur. Access points can also be used to provide access to other networks both wired (PSTN, xDSL etc.) and wireless (GSM, UMTS etc.).

Finally Bluetooth allows to realize low-cost and effective personal area networks. One typical scenario is a conference room where it would be possible to connect the video projector to the personal PC of the speaker and to record the (audio/video) compressed presentation in the attendant's PCs. PAN functionalities can find wide application in both domestic and office environments. Bluetooth devices enable the deployment of domotics services to render the environment intelligent and responsive to the different user needs. Another area for Bluetooth-based PANs is in the field of infomobility applications represent. In this case, one vehicle equipped with a PAN should be able to connect to any 
external network to gather any kind of information from the surrounding environment.

The basic unit of a Bluetooth network is the "piconet" and a simple scheme is drawn in Fig 1. Within one piconet up to eight active terminals can be connected

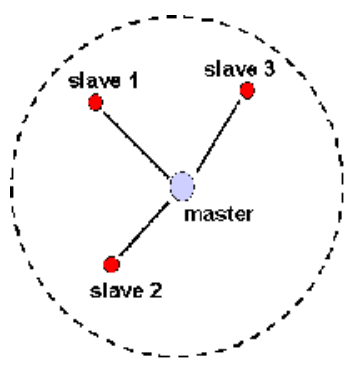

Fig. 1. Principle scheme of a piconet

and communicate over a common channel.

The modulation format is GFSK and when a piconet is created one participant assumes the master role and controls the piconet operations. The remaining units in the piconet are indicated as slaves and can communicate only with the master in a time division duplex (TDD) fashion according to a polling sequence. 1 Multiple piconets in the area can share the ISM band through a frequency hopping spread spectrum (FH-SS) scheme. A maximum of 79 hopping frequencies are considered. Each piconet is given a frequency hopping pattern which identifies the common channel used by the master and the slaves in the same piconet to communicate. The pattern is dictated by the master and slaves are synchronized with this hopping sequence. A piconet can contain an undefinite number of "parked" (i.e. not active) terminals that constantly maintain the synchronization with the master. A single Bluetooth unit can participate in more piconets, but it can serve as a master only in one piconet. A set of intercommunicating piconets is commonly referred to as a "scatternet". An example of a scatternet is illustrated in Fig 2 (a), where the roles of the different terminals in the three piconets have been depicted in Fig,2 (b).

\section{Bluetooth Technology: Market Status}

With the release of the set of Bluetooth specifications 1.0b the manufacturers started to produce and to market the first Bluetooth development kits but it was observed that Bluetooth systems produced by different manufacturers were not

\footnotetext{
${ }^{1}$ In the current standard no routing functionalities are provided by the master to provide connections between two slaves in the piconet. This is a subject of current research and the BNEP 4] profile seems to provide an effective solution to this problem.
} 


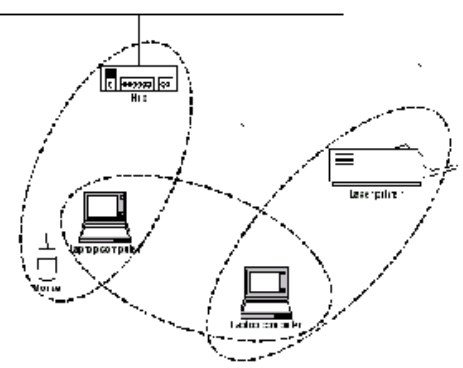

(a)

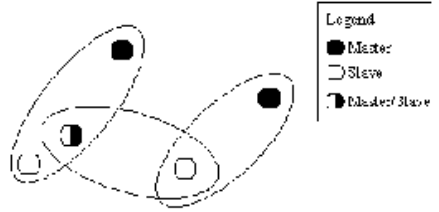

(b)

Fig. 2. Principle scheme of a scatternet - (a): architecture, (b): network topology

perfectly compatible and there were problems concerning the proposed inquiry and paging procedures. Many problems were solved with the set of specifications 1.1, [1] and to achieve a Bluetooth specification conformity the products of the different manufacturers need to comply with a set of tests defined by Bluetooth special interest group.

The typical Bluetooth development kit includes a Bluetooth chip-modem mounted on a board. The Bluetooth chip-modem can be connected to a host sending control commands and data through a number of standard interfaces such as RS232/UART, USB and JTAG.

The architecture of a generic Bluetooth chip-modem can be partitioned into two parts: an RF part and a baseband (BB) stage as shown in Fig.3. The BB performs co-decoding and link control operations.

The control of the Bluetooth modem by the host is achieved through a set of standardized packet commands defining the "so-called" host controller interface (HCI). A Bluetooth chip-modem BB stage contains all the hardware and software (i.e. firmware) to receive, to decode and to process the HCI commands.

To facilitate the communication of a Bluetooth chip-modem with software applications, a number of high level communication functions have been standardized and grouped into different protocol layers: L2CAP, RFCOMM, SDP etc. The lowest layers functions use the services offered by the HCI commands.

Manufacturers propose different solutions for the Bluetooth chip-modem based on one or on two chips implementations. The CSR (www.csr.com) BlueCore 01 is a single Bluetooth chip-modem containing both the RF and the BB stages in Fig 3. The firmware in the BB part can be updated and is able to execute the HCI commands only. Manufacturers such as Philips (www.philips.com), OKI (www.oki.com), GCT (www.gctsemi.com), SiliconWave (www.siliconwave.com), propose two chips solutions where the RF and the baseband stages are physically separated.

In both cases (single chip implementation or two chip implementation) the pins of the Bluetooth chip(s) are directly connected to standard interfaces such as RS232 or USB or JTAG. This greatly simplifies the creation of boards containing the Bluetooth chips.

A single chip solution allows to reduce system dimensions and power consumption. However, due to the integration of the RF and the BB stages on the same 


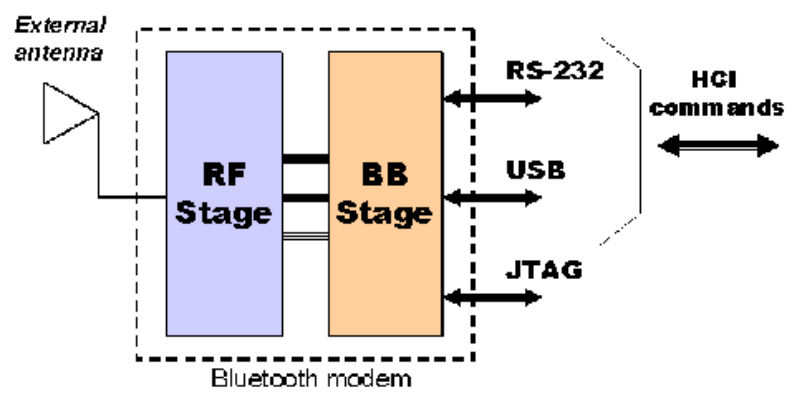

Fig. 3. architecture of a typical Bluetooth chip-modem

wafer the realization technology is much more expensive, thus increasing the cost of the final product. The adoption of separated processors allows to implement a more complex BB stage able to run also the desired application(s) as well as the upper layer protocols such as L2CAP, RFCOMM, SDP etc. This solution can be useful when a stand-alone Bluetooth embedded system is desired and the interfacing of the Bluetooth chip-modem with the device is completely controlled by the embedded system itself. However, as indicated by the CSR, the problems related to the scarce computation power in the baseband processor in the Bluecore 01 will be overcome in the next release of the chip indicated as Bluecore 02 so that it will be possible to implement a complete Bluetooth based system using a single chip.

\section{Packet Loss Probability in Bluetooth Networks}

Using different FH code patterns, several piconets can coexist in the same area indicated as served area, realizing a Bluetooth network The terminals aggregate randomly to form a large number of uncoordinated piconets with a different number of slaves. In a Bluetooth network packet collisions may occur when different piconets are transmitting simultaneously on the same frequency. This leads to an interference in the receiver (possibly) causing packet loss. Therefore, the packet loss probability (PLP) is an important performance index. PLP calculation needs to account for the dependency of the packet interference on the spatial distribution of terminals and on the environment characteristics. Results on this topic have been already presented in the literature [7]-9. An analytical approach for the PLP calculation was presented in [8], but results were restricted to a three overlapping piconets and a simple propagation model. In [9] a PLP upper bound is given without considering the "mitigation" effects due to propagation losses. In this section we provide a closed form expression for the PLP able to account for the geometry of the environment, its propagation characteristics and for the position of the reference receiver ( $\mathrm{RR})$.

We consider a Bluetooth network with $M+1$ piconets. The PLP is commonly defined as the probability that the signal to interference plus noise ratio at the 
output of the RR falls below a threshold, $\rho_{0}$, which accounts for the fast fading characteristics of the environment:

$$
P_{L P}(M)=\operatorname{Prob}\left\{\frac{C}{\sum_{m=1}^{M} \chi_{m} Y_{m}+N} \leq \rho_{0},\right\}
$$

where $C$ is the received power at the RR, $N$ is the noise power. The $\sum_{m=1}^{M} \chi_{m} Y_{m}$ is the total interference power where $Y_{m}$ is the interference power from the $m$-th piconet and $\chi_{m}$ is a binary random variable assuming with probability $q_{m}=1-p_{m}$. The $p_{m}$ is the probability that the $m$-th piconet in the area transmits on the same frequency slot of the RR. Consider only one-slot packet transmission and assume that each slot always contains a packet. For the case in which packet duration is equal to the time slot, we have:

$$
p_{m}=p=\left\{\begin{array}{cl}
1 / N_{f}, & \text { syncronized piconets } \\
1-\left(1-1 / N_{f}\right)^{2} & \text { assyncronized piconets }
\end{array},\right.
$$

where $N_{f}$ is the number of hopping frequencies $\left(N_{f}=79\right)$. Other expressions of $p$ in (2) for different values of the packet duration compared to the time slot are reported in [9].

The $C$ and $Y_{m}$ depends on the propagation losses due to the transmitter-receiver distance $d$ and to the obstacles geometry. Therefore, indicating with $W_{T}$ the terminal transmitted power assumed to be constant (i.e. no power control) we have: $C=W_{T} \times L_{c}$, and $Y_{m}=W_{T} \times L_{m}$. 2 The power losses $L_{c}, L_{m}$ depend on the position of the RR and of the $m$-th interferer. When a stochastic propagation model is considered, $L_{m}$ can be factored into two components, i.e. $L_{m}=A_{m} \times$ $R_{m}$, where $A_{m}$ is a deterministic component usually referred to as path loss and depends on the transmitter-receiver distance $d ; R_{m}$ is a random component accounting for shadowing. Since the power transmitted by each interfering user goes through the same propagation environment, the statistics of the interfering power measured at the RR are independent on $m$. Therefore, in the following we omit the dependency on $m$ and we consider $Y=W_{T} \times L$. We restrict our analysis to a 2-D environment. Both $C$ and $Y$ are considered as random variables with probability density functions (p.d.f.) $f_{C}(x)$ and $f_{Y}(x)$ that in general depend on the position of the RR in the area. They can be evaluated as indicated in 10 using numerical approximations provided the statistics of $A=A_{m}, R=R_{m}$ and spatial distribution of the interfering terminals in the served area, are given. From simple algebraic manipulations, equation (1) can be rewritten as:

$$
P_{L P}(M)=\operatorname{Prob}\left\{Z_{M} \leq 0\right\},
$$

with $Z_{M}=C-\rho_{0} N-\rho_{0} \sum_{m=1}^{M} \chi_{m} Y_{m}=Z_{M-1}-\varepsilon_{M}$ where $\varepsilon_{M}=\rho_{0} \chi_{M} Y_{M}$ and $Z_{0}=C-\rho_{0} N$. For simplicity but without loss of generality in the following derivation we omit the noise power $N$. From (3) the p.d.f. of $Z_{M}$ is:

$$
f_{Z_{M}}(x)=f_{C}(x) \otimes f_{\varepsilon_{1}}(-x) \otimes \cdots \otimes f_{\varepsilon_{M}}(-x),
$$

\footnotetext{
${ }^{2}$ No power control assumption is not valid for Bluetooth class 1 devices where power control is mandatory [1]
} 
with $f_{Z_{0}}(x)=f_{C}(x)$. After some calculations (3) can be rewritten in a more compact form as:

$$
P_{L P}(M)=\sum_{m=1}^{M}\left(\begin{array}{c}
M \\
m
\end{array}\right) q^{M-m} p^{m} \beta_{m}
$$

where $\beta_{m}=\int_{-\infty}^{0} g_{m}(x) \otimes f_{Z_{0}}(x) d x$ and $g_{m}(x)=\rho_{0}^{-m} f_{Y_{1}}\left(-x / \rho_{0}\right) \otimes \cdots \otimes$ $f_{Y_{M}}\left(-x / \rho_{0}\right)$ for $m=1,2, \ldots, M$ and $g_{0}(x)=\delta(x)$. The coefficient $\left(\begin{array}{c}M \\ m\end{array}\right) q^{M-m} p^{m}$ is the probability that $m$ among the $M$ interfering piconets are transmitting on the same frequency of the RR. The coefficients $\beta_{m}$ in (5) account for the PLP reduction due to path loss and shadowing. In fact, it is straightforward to observe that $\beta_{m}$ is always less than one for each $m$ and increases with $m$ approaching to one as $m$ tends to infinity. In addition the coefficients $\beta_{m}$ depend on the position of the RR and on the dimensions of the network area compared to the RR coverage area.

In the simple case of $\beta_{m}=1$ for each $m$ we obtain the upper bound reported in [9] i.e.:

$$
P_{L P}(M)=\sum_{m=1}^{M}\left(\begin{array}{c}
M \\
m
\end{array}\right) q^{M-m} p^{m} \beta_{m} \leq 1-q^{M} .
$$

Equation (5) can be conveniently rewritten as:

$$
P_{L P}(M)=1-q^{M}-\sum_{m=1}^{M}\left(\begin{array}{c}
M \\
m
\end{array}\right) q^{M-m} p^{m}\left(1-\beta_{m}\right) .
$$

Equation (7) allows to obtain successive approximations by neglecting the terms corresponding to $\beta_{m}$ close to unity. This can be useful for large $M$ when it can be difficult to obtain a good numerical approximation of $\beta_{m}$.

\section{Bluetooth Network Aggregate Throughput}

In the definition of the aggregate network throughput we need to account for the variations of the PLP with the position of the RR. We assume that piconets in the area have a number of units $L$ and that fixed length packets are used for transmission (for example DM1); the slaves are always transmitting towards the master in their time slots and the master has always something to transmit for all slaves; a round-robin baseband scheduling is considered and no signalling information is exchanged over the radio interface. On the basis of the previous assumptions, the master uses the $50 \%$ of the radio resource whereas the other $50 \%$ is fair shared among the $L-1$ slaves. Hence, indicating with $C$ the overall radio capacity in the piconet measured in packet per seconds, in an error free environment the master gets $C / 2$, and each slave gets $C /(2(L-1))$.

Now, we focus our attention on the throughput of a master/slave bidirectional connection in the presence of PLP and assuming $M$ piconets in the area. We 
define the throughput of a single connection of the piconet as the mean number of packets successfully received in the unit time. In this case considering the master to slave direction, it is easy to prove the overall throughput $\left(T_{M S}\right)$ in the piconet:

$$
T_{M S}\left(M, x_{1}, y_{1}, \ldots, x_{L-1}, y_{L-1}, L\right)=\frac{C}{2(L-1)} \sum_{l=1}^{L-1}\left(1-P_{L P}\left(x_{i}, y_{i}, M\right)\right)
$$

where $\left(x_{i}, y_{i}\right)$ indicates the coordinates of the slaves and the dependence of the PLP on $\left(x_{i}, y_{i}\right)$ has been evidenced.

Due to the Bluetooth mechanisms, a slave is allowed to transmit to the master only when it receives a packet from the master. So, when a master packet is lost, the return slot is wasted. Taking into account for this effect, the overall throughput of the piconet in the slave master direction can be written as:

$$
\begin{gathered}
T_{S M}\left(M, x_{1}, y_{1}, \ldots, x_{L-1}, y_{L-1}, X_{M}, Y_{M}, L\right)= \\
=\frac{C}{2(L-1)} \sum_{l=1}^{L-1}\left(1-P_{L P}\left(x_{i}, y_{i}, M\right)\right)\left(1-P_{L P}\left(X_{m}, Y_{m}, M\right)\right) .
\end{gathered}
$$

where $\left(X_{m}, Y_{m}\right)$ indicate the master coordinates. Hence, the total throughput of the piconet is:

$$
T_{P}\left(M, x_{1}, y_{1}, \ldots, x_{L-1}, y_{L-1}, X_{M}, Y_{M}, L\right)=T_{M S}+T_{S M},
$$

Fixing the position $(X m, Y m)$ of the master in the area and assuming that the slaves coordinates are randomly generated independently we can define the average throughput of the piconet as:

$$
\bar{T}_{P}\left(M, X_{M}, Y_{M}\right)=\int T_{P} \prod_{l=1}^{L-1} g\left(x_{l}, y_{l} \mid X_{m}, Y_{m}\right) d x_{l} d y_{l}
$$

where the integral is extended to the master coverage area and $g\left(x_{i}, y_{i} \mid X_{m}, Y_{m}\right)$ $=g\left(x_{1}, y_{1} \mid X_{m}, Y_{m}\right)$ with $i=2, \ldots, L-1$ is the probability density function of the slave coordinates given the master position. Due to the statistical independence of the slave coordinates using (10) it can be shown after some algebraic manipulations that (11) is independent on the number of slaves in the piconet. Considering $M$ piconets in the served area we can define the average aggregate network throughput as:

$$
T_{N}(M)=\int \bar{T}_{P}\left(M, X_{M}, Y_{M}\right) f\left(X_{m}, Y_{m}\right) d X_{m} d Y_{m}
$$

where the integral in (12) is extended to the served area and $f\left(X_{m}, Y_{m}\right)$ is the p.d.f. of the master coordinates. Assuming a uniform distribution we have $f\left(X_{m}, Y_{m}\right)=M / S_{a}$ where $S_{a}$ is the surface of the served area. The calculation of (12) is dependent on the environment characteristics of the served area not 
allowing to outiline general conclusions. Therefore, we resort to simple upper and lower bounds to give an idea on the throughput variability. Indicating with $P_{\min }(M)$ and $P_{\max }(M)$ the minimum and the maximum PLP in the served area, using these values in (10), we obtain:

$$
T_{N}(M) \geq\left(M C\left(1-P_{\max }(M)\right)\left(1-\frac{P_{\max }(M)}{2}\right)\right.
$$

and

$$
T_{N}(M) \leq\left(M C\left(1-P_{\min }(M)\right)\left(1-\frac{P_{\min }(M)}{2}\right)\right.
$$

\section{$6 \quad$ Numerical Results}

\subsection{Validation of the Proposed Approach}

To assess the effectiveness of equation (5) we performed a Monte Carlo simulation considering a typical operating scenario. In each trial we generate $M$ masters uniformly located in a rectangular served area. Each master forms a piconet with $N_{s}$ active slaves, where $N_{s}$ is a random number, uniformly distributed between 1 to 7 . Following the recommendations in [5], the transmitted power $W_{T}$ is set to $0 \mathrm{dBm}$. We assumed the following dual slope model for path loss, [1]:

$$
A(d)=\left\{\begin{array}{c}
40+20 \log _{10}(d), \quad d \leq 8.5 m \\
25.3+36 \log _{10}(d), d>8.5 m
\end{array}\right.
$$

To simplify the calculation procedure we neglected the presence of noise and shadowing. Assuming a receiver sensitivity of $-70 \mathrm{dBm}$ using (8), the RR coverage area is circular with radius $10 \mathrm{~m}$ [2]. The $N_{s}$ slaves participating in a piconet are randomly located according to a uniform distribution, in a circular area of 20 meters diameter, centered in the position of the piconet master. In generating the slaves' positions we ensured that they were confined within the served area. The transmitter to the $\mathrm{RR}$ is randomly located in a circular coverage area centered in the $\mathrm{RR}$ with $10 \mathrm{~m}$ radius. In this case when the piconets interference is null we assume that no packet loss occurs. Each piconet transmission begins in a randomly selected time slot. In every piconet the master begins the transmission by sending an ACL packet to one of the $N_{s}$ slaves belonging to its piconet. For each master we randomly generated its own channel hopping sequence assuming a uniform distribution over the 79 frequency carriers. The length of the frequency hopping sequence for each master was taken equal to the duration of the simulation trial. We averaged the performance metrics over a large number of simulation trials for each scenario. In each trail we changed the users' positions and the piconet loads (the number of slaves in each piconet). In each time slot we compute the signal-to-interference ratio $(C / I)$ of the RR. In Fig.4 we compare the $P_{L P}(M)$ obtained from (5) with the results of a Monte Carlo simulation obtained for different dimensions of the served area supposed 


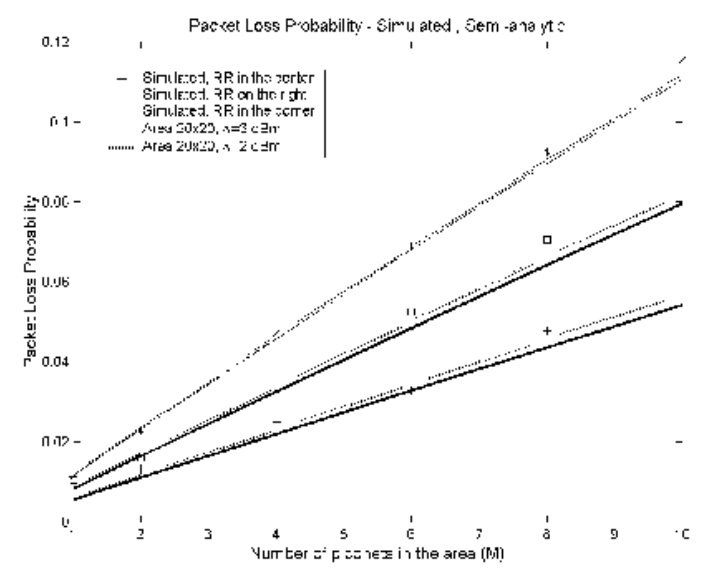

Fig. 4. Packet Loss Probability vs the number of piconets in the area - Area dimensions: $20 \times 20 \mathrm{~m}$ - synchronous piconets

to be rectangular and for different positions of the RR. Discrete numerical approximation of the p.d.fs. $f_{C}(x)$ and $f_{Y}(x)$ were used to obtain the coefficients $\beta_{m}$ in (5) (see [10]). The discrete step $\Delta$ of the random variable $x$ is indicated in Fig.4. As expected the agreement between the simulated and theoretical results improves reducing $\Delta$ at the expense of an increased computation time. From the results in Fig.4 it can be observed that PLP significantly changes with the position of the RR in the served area.

\subsection{Network Throughput Calculation}

We consider a rectangular served area, and the propagation model in (15). In this case it is straightforward to observe that the minimum PLP is obtained when the $\mathrm{RR}$ is placed in the corner and the maximum PLP is obtained when the RR is placed in the center. We introduce the normalized aggregate throughput $S(M)$ defined as $S(M)=T_{N}(M) / C$. In Fig.5 we plot the upper and lower bounds of the normalized scatternet throughput as a function of the number of piconets and for different dimensions of the served area. To evaluate the PLP we used equation (5) and the corresponding values of $\beta_{m}$ are reported in Table 1. To obtain the results in Fig [5] and in Table 1 a uniform distribution of the interferers in the area was assumed.

From Fig $[5$ we observe that increasing in the number of piconets, the average normalized Bluetooth network throughput increases as well until a critical value of piconets is reached. Over this value the interference due to the higher number of collisions reduces the overall throughput. When the area is small compared to the coverage area of the RR, it is possible to adopt the PLP upper bound in (6) to evaluate the network throughput. 
Table 1. Values of $\beta_{m}$ used in [5) - RR in the center and in the corner of the served area

\begin{tabular}{lcccccc}
\hline \multirow{2}{*}{$\mathbf{m} /$ Area } & \multicolumn{2}{c}{$\mathbf{1 0 m} \times \mathbf{1 0 m}$} & \multicolumn{2}{c}{$\mathbf{2 0 m} \times \mathbf{2 0 m}$} & \multicolumn{2}{c}{$\mathbf{2 0 m} \times \mathbf{1 0 m}$} \\
& Center & Corner & Center & Corner & Center & Corner \\
\hline$\beta_{1}$ & 0.9449 & 0.9115 & 0.9112 & 0.4020 & 0.9238 & 0.6208 \\
$\beta_{2}$ & 0.9848 & 0.9770 & 0.9771 & 0.7683 & 0.9802 & 0.8979 \\
$\beta_{3}$ & 0.9895 & 0.9855 & 0.9856 & 0.8665 & 0.9877 & 0.9479 \\
$\beta_{4}$ & 0.9931 & 0.9903 & 0.9904 & 0.9209 & 0.9917 & 0.9695 \\
$\beta_{5}$ & 0.9947 & 0.9922 & 0.9923 & 0.9449 & 0.9934 & 0.9823 \\
$\beta_{6}$ & 0.9975 & 0.9952 & 0.9948 & 0.9566 & 0.9965 & 0.9902 \\
\hline
\end{tabular}

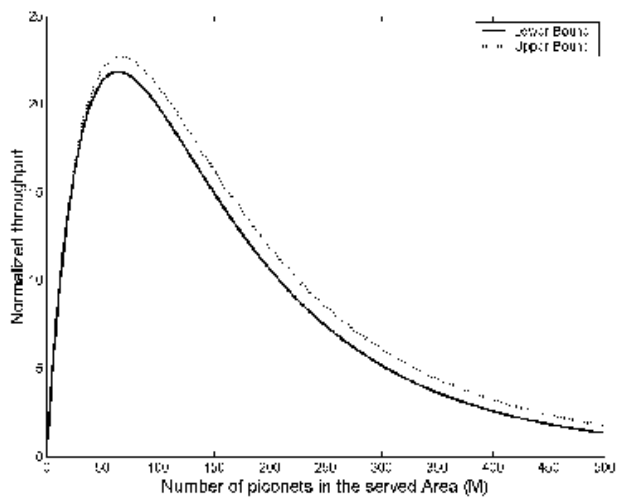

(a)

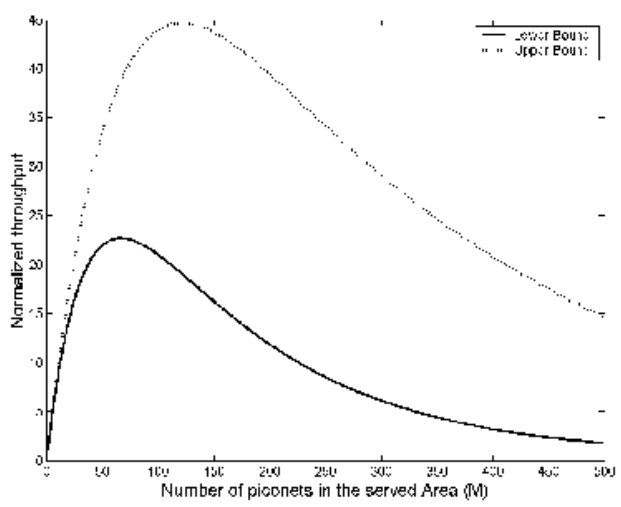

(b)

Fig. 5. Normalized Bluetooth network throughput vs the number of piconets - upper and lower bounds area dimensions: (a): 10mx10m, (b): $20 \mathrm{mx} 20 \mathrm{~m}$ 
This is no longer true for large areas where the difference between the upper and lower bounds of the throughput can be relevant (see Fig.5b) since the beneficial effects due to environments cannot be neglected.

\section{Conclusions}

The Bluetooth transmission technology is a viable and low cost solution to fulfill many of the requirements of short range wireless communications. In this paper we reviewed the technological aspects of the Bluetooth products available on the market and we discussed the advantages and drawbacks of one or two chips implementation. We analyzed the performance of a Bluetooth network consisting of a set of uncoordinated Bluetooth terminals in the same area in terms of PLP and aggregate network throughput. We obtained a closed form expression for the PLP accounting for the beneficial effects due to propagation losses. The effectiveness of the proposed calculation was assessed through a comparison with simulation results. As expected the PLP is strongly dependent on the position of the $\mathrm{RR}$ and on the size of the served area compared to the coverage area. Large variations of the upper and lower bounds of the network aggregate throughput with the PLP have been evidenced.

\section{References}

1. Specification of the Bluetooth System: Core, Version 1.1. February 22, 2001.

2. J. C. Haartsen, S. Mattison, "Bluetooth-A New Low-Power Radio Interface Providing Short-Range Connectivity", Proc. IEEE Vol. 88, No. 10, October 2000, pp.1651-1661

3. Jaap C. Haartsen, "The Bluetooth Radio System", IEEE Pers. Comm., February 2000, pp.28-36

4. Official Bluetooth website: http://www.bluetooth.com

5. P. Johansson, M. Kazantzidis, R. Kapoor, M. Gerla, "Bluetooth: an enabler for personal area networking", IEEE Network, Vol. 15, Issue: 5 Sept.-Oct. 2001, pp. 28-37

6. Bhagwat, P. "Bluetooth: Technology for Short-range Wireless Applications", IEEE Internet Computing, Volume: 5 Issue: 3 , May-June 2001 Page(s): 96 -103

7. Y. Lim, J. Kim, S. L. Min and J. Soo Ma, "Performance evaluation of the Bluetooth-based public internet access point," IEEE, 2001

8. A. Karnik and A. Kumar, "Performance analysis of the Bluetooth physical layer," Proceedings of the IEEE ICPWC, 2000.

9. A. El-Hoiydi, "Interference between Bluetooth Networks - upper bound on the packet error rate," IEEE Commun. Lett., vol. 5, No. 6, June 2001

10. F. Mazzenga, D. Cassioli, P. Loreti, F. Vatalaro, "Evaluation of Packet Loss Probability in Bluetooth Networks", to be presented in ICC 2002, April 28- May 2, 2002, New York City, USA 